\title{
Oriented diameter and rainbow connection number of a graph $\|^{*}$
}

\author{
Xiaolong Huang Hengzhe Li $\|^{\dagger} \quad$ Xueliang Li Yuefang Sun
}

Center for Combinatorics and LPMC-TJKLC, Nankai University, Tianjin, China

received 23rd Dec. 2012, accepted 28 $8^{\text {th }}$ May 2014.

The oriented diameter of a bridgeless graph $G$ is $\min \{\operatorname{diam}(H) \mid H$ is a strang orientation of $G\}$. A path in an edge-colored graph $G$, where adjacent edges may have the same color, is called rainbow if no two edges of the path are colored the same. The rainbow connection number $\operatorname{rc}(G)$ of $G$ is the smallest integer number $k$ for which there exists a $k$-edge-coloring of $G$ such that every two distinct vertices of $G$ are connected by a rainbow path. In this paper, we obtain upper bounds for the oriented diameter and the rainbow connection number of a graph in terms of $\operatorname{rad}(G)$ and $\eta(G)$, where $\operatorname{rad}(G)$ is the radius of $G$ and $\eta(G)$ is the smallest integer number such that every edge of $G$ is contained in a cycle of length at most $\eta(G)$. We also obtain constant bounds of the oriented diameter and the rainbow connection number for a (bipartite) graph $G$ in terms of the minimum degree of $G$.

Keywords: Diameter, Radius, Oriented diameter, Rainbow connection number, Cycle length, Bipartite graph.

\section{Introduction}

All graphs in this paper are undirected, finite and simple. We refer to the book [2] for notation and terminology not described here. A path $u=u_{1}, u_{2}, \ldots, u_{k}=v$ is called a $P_{u, v}$ path. Denote by $u_{i} P u_{j}$ the subpath $u_{i}, u_{i+1}, \ldots, u_{j}$ for $i \leq j$. The length $\ell(P)$ of a path $P$ is the number of edges in $P$. The distance between two vertices $x$ and $y$ in $G$, denoted by $d_{G}(x, y)$, is the length of a shortest path between them. The eccentricity of a vertex $x$ in $G$ is $\operatorname{ecc}_{G}(x)=\max _{y \in V(G)} d(x, y)$. The radius and diameter of $G$ are $\operatorname{rad}(G)=\min _{x \in V(G)} \operatorname{ecc}(x)$ and $\operatorname{diam}(G)=\max _{x \in V(G)} \operatorname{ecc}(x)$, respectively. A vertex $u$ is a center of a graph $G$ if $\operatorname{ecc}(u)=\operatorname{rad}(G)$. The oriented diameter of a bridgeless graph $G$ is $\min \{\operatorname{diam}(H) \mid H$ is an orientation of $G\}$, and the oriented radius of a bridgeless graph $G$ is $\min \{\operatorname{rad}(H) \mid H$ is an orientation of $G\}$. For any graph $G$ with edge-connectivity $\lambda(G)=0,1, G$ has oriented radius (resp. diameter) $\infty$.

In 1939, Robbins solved the One-Way Street Problem and proved that a graph $G$ admits a strongly connected orientation if and only if $G$ is bridgeless, that is, $G$ does not have any cut-edge. Naturally, one hopes that the oriented diameter of a bridgeless graph is as small as possible. Bondy and Murty suggested

*Supported by NSFC No.11071130, and "the Fundamental Research Funds for the Central Universities".

†Email: Ihz2010@mail.nankai.edu.cn, lhz@htu.cn 
to study the quantitative variations on Robbins' theorem. In particular, they conjectured that there exists a function $f$ such that every bridgeless graph with diameter $d$ admits an orientation of diameter at most $f(d)$.

In 1978, Chvátal and Thomassen [5] obtained some general bounds.

Theorem 1 (Chvátal and Thomassen 1978 [5]) For every bridgeless graph $G$, there exists an orientation $H$ of $G$ such that

$$
\begin{aligned}
\operatorname{rad}(H) & \leq \operatorname{rad}(G)^{2}+\operatorname{rad}(G), \\
\operatorname{diam}(H) & \leq 2 \operatorname{rad}(G)^{2}+2 \operatorname{rad}(G) .
\end{aligned}
$$

Moreover, the above bounds are optimal.

There exists a minor error when they constructed the graph $G_{d}$ which arrives at the upper bound when $d$ is odd. Kwok, Liu and West gave a slight correction in [11].

They also showed that determining whether an arbitrary graph can be oriented so that its diameter is at most 2 is NP-complete. Bounds for the oriented diameter of graphs have also been studied in terms of other parameters, for example, radius, dominating number [5, 6, 11, 18], etc. Some classes of graphs have also been studied in [6, 7, 8, 9, 14].

Let $\eta(G)$ be the smallest integer such that every edge of $G$ belongs to a cycle of length at most $\eta(G)$. In this paper, we show the following result.

Theorem 2 For every bridgeless graph $G$, there exists an orientation $H$ of $G$ such that

$$
\begin{gathered}
\operatorname{rad}(H) \leq \sum_{i=1}^{\operatorname{rad}(G)} \min \{2 i, \eta(G)-1\} \leq \operatorname{rad}(G)(\eta(G)-1), \\
\operatorname{diam}(H) \leq 2 \sum_{i=1}^{\operatorname{rad}(G)} \min \{2 i, \eta(G)-1\} \leq 2 \operatorname{rad}(G)(\eta(G)-1) .
\end{gathered}
$$

Note that $\sum_{i=1}^{\operatorname{rad}(G)} \min \{2 i, \eta(G)-1\} \leq \operatorname{rad}(G)^{2}+\operatorname{rad}(G)$ and $\operatorname{diam}(H) \leq 2 \operatorname{rad}(H)$. So our result implies Chvátal and Thomassen's Theorem 1

A path in an edge-colored graph $G$, where adjacent edges may have the same color, is called rainbow if no two edges of the path are colored the same. An edge-coloring of a graph $G$ is a rainbow edge-coloring if every two distinct vertices of the graph $G$ are connected by a rainbow path. The rainbow connection number $\operatorname{rc}(G)$ of $G$ is the minimum integer $k$ for which there exists a rainbow $k$-edge-coloring of $G$. It is easy to see that $\operatorname{diam}(G) \leq r c(G)$ for any connected graph $G$. The rainbow connection number was introduced by Chartrand et al. in [4]. It is of great use in transferring information of high security in multicomputer networks. We refer the readers to [3] for details.

Chakraborty et al. [3] investigated the hardness and algorithms for the rainbow connection number, and showed that given a graph $G$, deciding if $r c(G)=2$ is $N P$-complete. Bounds for the rainbow connection number of a graph have also been studies in terms of other graph parameters, for example, radius, dominating number, minimum degree, connectivity, etc. [1, 4, 10]. Cayley graphs and line graphs were studied in [12] and [13], respectively.

A subgraph $H$ of a graph $G$ is called isometric if the distance between any two distinct vertices in $H$ is the same as their distance in $G$. The size of a largest isometric cycle in $G$ is denoted by $\zeta(G)$. 
Clearly, every isometric cycle is an induced cycle and thus $\zeta(G)$ is not larger than the chordality, where chordality is the length of a largest induced cycle in $G$. In [1], Basavaraju, Chandran, Rajendraprasad and Ramaswamy got the the following sharp upper bound for the rainbow connection number of a bridgeless graph $G$ in terms of $\operatorname{rad}(G)$ and $\zeta(G)$.

Theorem 3 (Basavaraju et al. [1]) For every bridgeless graph $G$,

$$
r c(G) \leq \sum_{i=1}^{\operatorname{rad}(G)} \min \{2 i+1, \zeta(G)\} \leq \operatorname{rad}(G) \zeta(G) .
$$

In this paper, we show the following result.

Theorem 4 For every bridgeless graph $G$,

$$
r c(G) \leq \sum_{i=1}^{\operatorname{rad}(G)} \min \{2 i+1, \eta(G)\} \leq \operatorname{rad}(G) \eta(G) .
$$

From Lemma 2 of Section 2, we will see that $\eta(G) \leq \zeta(G)$. Thus our result implies Theorem 3

This paper is organized as follows: in Section 2, we introduce some new definitions and show several lemmas. In Section 3, we prove Theorem 2 and study upper bounds for the oriented radius (resp. diameter) of plane graphs, edge-transitive graphs and general (bipartite) graphs. In Section 4, we prove Theorem 4 and study upper for the rainbow connection number of plane graphs, edge-transitive graphs and general (bipartite) graphs.

\section{Preliminaries}

In this section, we introduce some definitions and show several lemmas.

Definition 1 For any $x \in V(G)$ and $k \geq 0$, the $k$-step open neighborhood is $\{y \mid d(x, y)=k\}$ and denoted by $N_{k}(x)$, the $k$-step closed neighborhood is $\{y \mid d(x, y) \leq k\}$ and denoted by $N_{k}[x]$. If $k=1$, we simply write $N(x)$ and $N[x]$ for $N_{1}(x)$ and $N_{1}[x]$, respectively.

Definition 2 Let $G$ be a graph and $H$ be a subset of $V(G)$ (or a subgraph of $G$ ). The edges between $H$ and $G \backslash H$ are called legs of $H$. An $H$-ear is a path $P=\left(u_{0}, u_{1}, \ldots, u_{k}\right)$ in $G$ such that $V(H) \cap V(P)=$ $\left\{u_{0}, u_{k}\right\}$. The vertices $u_{0}, u_{k}$ are called the feet of $P$ in $H$ and $u_{0} u_{1}, u_{k-1} u_{k}$ are called the legs of $P$. The length of an $H$-ear is the length of the corresponding path. If $u_{0}=u_{k}$, then $P$ is called a closed $H$ ear. For any leg $e$ of $H$, denote by $\ell(e)$ the smallest number such that there exists an $H$-ear of length $\ell(e)$ containing $e$, and such an $H$-ear is called an optimal $(H, e)$-ear.

Note that for any optimal $(H, e)$-ear $P$ and every pair $(x, y) \neq\left(u_{0}, u_{k}\right)$ of distinct vertices of $P, x$ and $y$ are adjacent on $P$ if and only if $x$ and $y$ are adjacent in $G$.

Definition 3 For any two paths $P$ and $Q$, the joint of $P$ and $Q$ are the common vertex and edge of $P$ and $Q$. Paths $P$ and $Q$ have $k$ continuous common segments if the common vertex and edge are $k$ disjoint paths.

A common segment is trivial if it has only one vertex. 
Definition 4 Let $P$ and $Q$ be two paths in $G$. Call $P$ and $Q$ independent if they has no common internal vertex.

Lemma 1 Let $n \geq 1$ be an integer, and let $G$ be a graph, $H$ be a subgraph of $G$ and $e_{i}=u_{i} v_{i}$ be a leg of $H$ and $P_{i}=P_{u_{i} w_{i}}$ be an optimal $\left(G, e_{i}\right)$-ear, where $1 \leq i \leq n$ and $u_{i}, w_{i}$ are the foot of $P_{i}$. Then for any leg $e_{j}=u_{j} v_{j}$ such that $e_{j} \neq e_{i}$ and $e_{j} \notin E\left(P_{i}\right)$, where $i \in\{1,2, \ldots, n\}$, there exists an optimal $\left(H, e_{j}\right)$-ear $P_{j}=P_{u_{j} w_{j}}$ such that either $P_{i}$ and $P_{j}$ are independent for any $P_{i}, 1 \leq i \leq n$, or $P_{i}$ and $P_{j}$ have only one continuous common segment containing $w_{j}$ for some $P_{i}$.

Proof: Let $P_{j}$ be an optimal $\left(H, e_{j}\right)$-ear. If $P_{i}$ and $P_{j}$ are independent for any $i$, then we are done. Suppose that $P_{i}$ and $P_{j}$ have $m$ continuous common segments for some $i$, where $m \geq 1$. When $m \geq 2$, we first

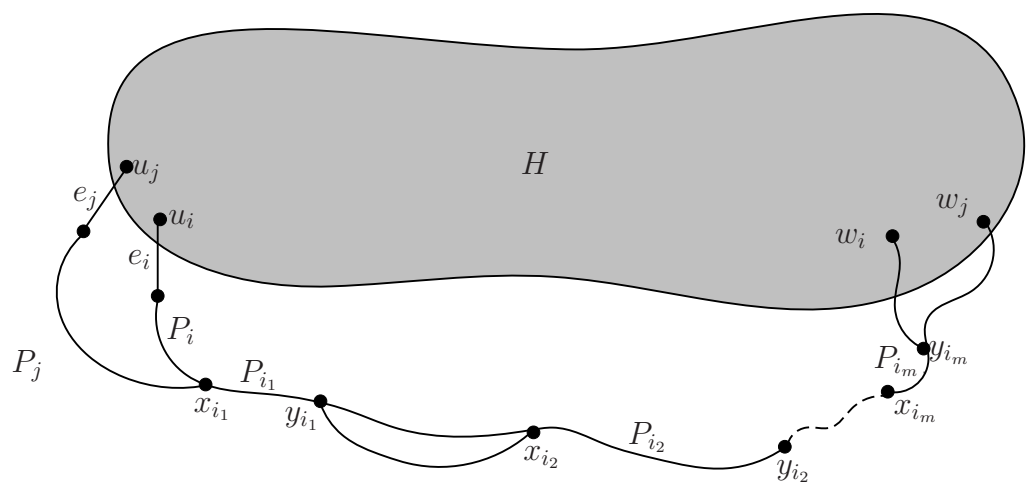

Fig. 1: Two $H$-ears $P_{i}$ and $P_{j}$

construct an optimal $\left(H, e_{j}\right)$-ear $P_{j}^{*}$ such that $P_{i}$ and $P_{j}^{*}$ has only one continuous common segment. Let $P_{i_{1}}, P_{i_{2}}, \ldots, P_{i_{m}}$ be the $m$ continuous common segments of $P_{i}$ and $P_{j}$ and they appear in $P_{i}$ in that order. See Figure 1 for details. Furthermore, suppose that $x_{i_{k}}$ and $y_{i_{k}}$ are the two ends of the path $P_{i_{k}}$ and they appear in $P_{i}$ successively. We say that the following claim holds.

Claim 1: $\ell\left(y_{k} P_{i} x_{k+1}\right)=\ell\left(y_{k} P_{j} x_{k+1}\right)$ for any $1 \leq k \leq m-1$.

If not, that is, there exists an integer $k$ such that $\ell\left(y_{k} P_{i} x_{k+1}\right) \neq \ell\left(y_{k} P_{j} x_{k+1}\right)$. Without loss of generality, we assume $\ell\left(y_{k} P_{i} x_{k+1}\right)<\ell\left(y_{k} P_{j} x_{k+1}\right)$. Then we shall get a more shorter path $H$-ear containing $e_{j}$ by replacing $y_{k} P_{j} x_{k+1}$ with $y_{k} P_{i} x_{k+1}$, a contradiction. Thus $\ell\left(y_{k} P_{i} x_{k+1}\right)=\ell\left(y_{k} P_{j} x_{k+1}\right)$ for any $k$.

Let $P_{j}^{*}$ be the path obtained from $P_{j}$ by replacing $y_{k} P_{j} x_{k+1}$ with $y_{k} P_{i} x_{k+1}$, and let $P_{j}=P_{j}^{*}$. If the continuous common segment of $P_{i}$ and $P_{j}$ does not contain $w_{j}$. Suppose $x$ and $y$ are the two ends of the common segment such that $x$ and $y$ appeared on $P$ starting from $u_{i}$ to $w_{i}$ successively. Similar to Claim 1, $\ell\left(y P_{i} w_{i}\right)=\ell\left(y P_{j} w_{j}\right)$. Let $P_{j}^{*}$ be the path obtained from $P_{j}$ by replacing $y P_{j} w_{j}$ with $y P_{i} w_{i}$. Clearly, $P_{j}^{*}$ is our desired optimal $\left(H, u_{j} v_{j}\right)$-ear.

Lemma 2 For every bridgeless graph $G, \eta(G) \leq \zeta(G)$.

Proof: Suppose that there exists an edge $e$ such that the length $\ell(C)$ of the smallest cycle $C$ containing $e$ is larger than $\zeta(G)$. Then, $C$ is not an isometric cycle since the length of a largest isometric cycle is 
$\zeta(G)$. Thus there exist two vertices $u$ and $v$ on $C$ such that $d_{G}(u, v)<d_{C}(u, v)$. Let $P$ be a shortest path between $u$ and $v$ in $G$. Then a closed trial $C^{\prime}$ containing $e$ is obtained from the segment of $C$ containing $e$ between $u$ and $v$ by adding $P$. Clearly, the length $\ell\left(C^{\prime}\right)$ is less than $\ell(C)$. We can get a cycle $C^{\prime \prime}$ containing $e$ from $C^{\prime}$. Thus there exists a cycle $C^{\prime \prime}$ containing $e$ with length less than $\ell(C)$, a contradiction. Therefore $\eta(G) \leq \zeta(G)$.

Lemma 3 Let $G$ be a bridgeless graph and $u$ be a center of $G$. For any $i \leq \operatorname{rad}(G)-1$ and every leg $e$ of $N_{i}(u)$, there exists an optimal $\left(N_{i}[u], e\right)$-ear with length at most $\min \{2(\operatorname{rad}(G)-i)+1, \eta(G)\}$.

Proof: Let $P$ be an optimal $\left(N_{i}[u], e\right)$-ear. Since $e$ belongs to a cycle with length at most $\eta(G), \ell(P) \leq$ $\eta(G)$. On the other hand, if $\ell(P) \geq 2(\operatorname{rad}(G)-i)+1$, then the middle vertex of $P$ has distance at least $\operatorname{rad}(G)-i+1$ from $N_{i}[u]$, a contradiction.

\section{Oriented diameter}

At first, we have the following observation.

Observation 1 Let $G$ be a bridgeless graph and $H$ be a bridgeless spanning subgraph of $G$. Then the oriented radius (resp. diameter) of $G$ is not larger than the oriented radius (resp. diameter) of $H$.

Proof of Theorem 2; We only need to show that $G$ has an orientation $H$ such that

$$
\operatorname{rad}(H) \leq \sum_{i=1}^{\operatorname{rad}(G)} \min \{2 i, \eta(G)-1\} \leq \operatorname{rad}(G)(\eta(G)-1) .
$$

Let $u$ be a center of $G$ and let $H_{0}$ be the trivial graph with vertex set $\{u\}$. We assert that there exists $a$ subgraph $G_{i}$ of $G$ such that $N_{i}[u] \subseteq V\left(G_{i}\right)$ and $G_{i}$ has an orientation $H_{i}$ satisfying that $\operatorname{rad}\left(H_{i}\right) \leq$ $e c c_{H_{i}}(u) \leq \Sigma_{j=1}^{i} \min \{2(\operatorname{rad}(G)-j), \eta(G)-1\}$.

Basic step: When $i=1$, we omit the proof since the proof of this step is similar to that of the following induction step.

Induction step: Assume that the above assertion holds for $i-1$. Next we show that the above assertion also holds for $i$. For any $v \in N_{i}(u)$, either $v \in V\left(H_{i-1}\right)$ or $v \in N_{G}\left(V\left(H_{i-1}\right)\right)$ since $N_{i-1}[u] \subseteq$ $V\left(H_{i-1}\right)$. If $N_{i}(u) \subseteq V\left(H_{i-1}\right)$, then let $H_{i}=H_{i-1}$ and we are done. Thus, we suppose $N_{i}(u) \nsubseteq$ $V\left(H_{i-1}\right)$ in the following.

Let $X=N_{i}(u) \backslash V\left(H_{i-1}\right)$. Pick $x_{1} \in X$, let $y_{1}$ be a neighbor of $x_{1}$ in $H_{i-1}$ and let $P_{1}=P_{y_{1} z_{1}}$ be an optimal $\left(H_{i-1}, x_{1} y_{1}\right)$-ear. We orient $P$ such that $P_{1}$ is a directed path. Pick $x_{2} \in X$ satisfying that all incident edges of $x_{2}$ are not oriented. Let $y_{2}$ be a neighbor of $x_{2}$ in $H_{i-1}$. If there exists an optimal $\left(H_{i-1}, x_{2} y_{2}\right)$-ear $P_{2}$ such that $P_{1}$ and $P_{2}$ are independent, then we can orient $P_{2}$ such that $P_{2}$ is a directed path. Otherwise, by Lemma 1 there exists an optimal $\left(H_{i-1}, x_{2} y_{2}\right)$-ear $P_{2}=P_{y_{2} z_{2}}$ such that $P_{1}$ and $P_{2}$ has only one continuous common segment containing $z_{2}$. Clearly, we can orient the edges in $E\left(P_{2}\right) \backslash E\left(P_{1}\right)$ such that $P_{2}$ is a directed path. We can pick the vertices of $X$ and oriented optimal $H$-ears similar to the above method until that for any $x \in X$, at least two incident edges of $x$ are oriented. Let $H_{i}$ be the graph obtained from $H_{i-1}$ by adding vertices in $V(G) \backslash V\left(H_{i-1}\right)$, which has at least two new oriented incident edges, and adding the new oriented edges. Clearly, $N_{i}[u] \subseteq V\left(H_{i}\right)=V\left(G_{i}\right)$. 
Now we show that $\operatorname{rad}\left(H_{i}\right) \leq \Sigma_{j=1}^{i} \min \{2(\operatorname{rad}(G)-i), \eta(G)-1\}$. It suffices to show that for every vertex $x$ of $H_{i}, d_{H_{i}}\left(H_{i-1}, x\right) \leq \min \{2(\operatorname{rad}(G)-i), \eta(G)-1\}$ and $d_{H_{i}}\left(x, H_{i-1}\right) \leq \min \{2(\operatorname{rad}(G)-$ i), $\eta(G)-1\}$. If $x \in V\left(H_{i-1}\right)$, then the assertion holds by inductive hypothesis. If $x \notin V\left(H_{i-1}\right)$. Let $P$ be a directed optimal $\left(H_{i}, e\right)$-ear containing $x$, where $e$ is some leg of $H_{i-1}$ (such a leg and such an ear exists by the definition of $H_{i}$. By Lemma $3, \ell(P) \leq \min \{2(\operatorname{rad}(G)-i)+1, \eta(G)\}$. Thus, $d_{H_{i}}\left(x, H_{i-1}\right) \leq \min \{2(\operatorname{rad}(G)-i), \eta(G)-1\}$ and $d_{H_{i}}\left(H_{i-1}, x\right) \leq \min \{2(\operatorname{rad}(G)-i), \eta(G)-1\}$. Therefore, $\operatorname{rad}\left(H_{i}\right) \leq \sum_{j=1}^{i} \min \{2(\operatorname{rad}(G)-j), \eta(G)-1\}$.

Remark 1 The above theorem is optimal since it implies Chvátal and Thomassen's optimal Theorem 1. Readers can see [5] 11] for optimal examples.

The following example shows that our result is better than that of Theorem 1

Example 1 Let $F_{3}$ be a triangle with one of its vertices designated as a root. In order to construct $F_{r}$, take two copies of $F_{r-1}$. Let $H_{r}$ be the graph obtained from the triangle $u_{0}, u_{1}, u_{2}$ by identifying the root of first (resp. second) copy of $F_{r-1}$ with $u_{1}$ (resp. $u_{2}$ ), and $u_{0}$ be the root of $F_{r}$. Let $G_{r}$ be the graph obtained by taking two copies of $F_{r}$ and identifying their roots. See Figure 2 for details. It is easy to check that $G_{r}$ has radius $r$ and every edge belongs to a cycle of length $\eta(G)=3$. By Theorem 1 . $G_{r}$ has an orientation $H_{r}$ such that $\operatorname{rad}\left(H_{r}\right) \leq r^{2}+r$ and $\operatorname{diam}\left(H_{r}\right) \leq 2 r^{2}+2 r$. But, by Theorem 2 , $G_{r}$ has an orientation $H_{r}$ such that $\operatorname{rad}(G) \leq 2 r$ and $\operatorname{diam}(G) \leq 4 r$. On the other hand, it is easy to check that all the strong orientations of $G_{r}$ has radius $2 r$ and diameter $4 r$.

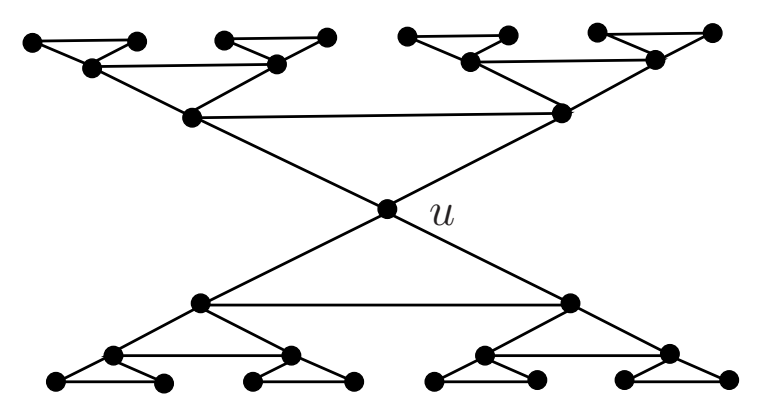

Fig. 2: The graph $G_{3}$ which has oriented radius 6 and oriented diameter 12

We have the following result for plane graphs.

Theorem 5 Let $G$ be a plane graph. If the length of the boundary of every face is at most $k$, then $G$ has an oriented $H$ such that $\operatorname{rad}(H) \leq \operatorname{rad}(G)(k-1)$ and diam $(H) \leq 2 \operatorname{rad}(G)(k-1)$.

Since every edge of a maximal plane (resp. outerplane) graph belongs to a cycle with length 3 , the following corollary holds.

Corollary 1 Let $G$ be a maximal plane (resp. outerplane) graph. Then there exists an orientation $H$ of $G$ such that $\operatorname{rad}(H) \leq 2 \operatorname{rad}(G)$ and $\operatorname{rad}(H) \leq 4 \operatorname{rad}(G)$.

A graph $G$ is edge-transitive if for any $e_{1}, e_{2} \in E(G)$, there exists an automorphism $g$ such that $g\left(e_{1}\right)=e_{2}$. We have the following result for edge-transitive graphs. 
Theorem 6 Let $G$ be a bridgeless edge-transitive graph. Then $G$ has an orientation $H$ such that rad $(H) \leq$ $\operatorname{rad}(G)(g(G)-1)$ and diam $(H) \leq 2 \operatorname{rad}(G)(g(G)-1)$, where $g(G)$ is the girth of $G$, that is, the length of a smallest induced cycle.

For general bipartite graphs, the following theorem holds.

Theorem 7 Let $G=\left(V_{1} \cup V_{2}, E\right)$ be a bipartite graph with $\left|V_{1}\right|=n$ and $\left|V_{2}\right|=m$. If $d(x) \geq k>\lceil m / 2\rceil$ for any $x \in V_{1}, d(y) \geq r>\lceil n / 2\rceil$ for any $y \in V_{2}$, then there exists an orientation $H$ of $G$ such that $\operatorname{rad}(H) \leq 9$.

Proof: It suffices to show that $\operatorname{rad}(G) \leq 3$ and $\eta(G) \leq 4$ by Theorem 2 .

First, we show that $\operatorname{rad}(G) \leq 3$. Fix a vertex $x$ in $G$, and let $y$ be any vertex different from $x$ in $G$. If $x$ and $y$ belong to the same part, without loss of generality, say $x, y \in V_{1}$. Let $X$ and $Y$ be neighborhoods of $x$ and $y$ in $V_{2}$, respectively. If $X \cap Y=\emptyset$, then $\left|V_{2}\right| \geq|X|+|Y| \geq 2 k>m$, a contradiction. Thus $X \cap Y \neq \emptyset$, that is, there exists a path between $x$ and $y$ of length two. If $x$ and $y$ belong to different parts, without loss of generality, say $x \in V_{1}, y \in V_{2}$. Suppose $x$ and $y$ are nonadjacent, otherwise there is nothing to prove. Let $X$ and $Y$ be the neighborhoods of $x$ and $y$ in $G$, and let $X^{\prime}$ be the set of neighbors of $X$ except for $x$ in $G$. If $X^{\prime} \cap Y=\emptyset$, then $\left|V_{1}\right| \geq 1+|Y|+\left|X^{\prime}\right| \geq 1+r+(r-1)=2 r>n$, a contradiction (Note that $\left|X^{\prime}\right| \geq r-1$ ). Thus $X^{\prime} \cap Y \neq \emptyset$, that is, there exists a path between $x$ and $y$ of length three in $G$.

Next we show that $\eta(G) \leq 4$. Let $x y$ be any edge in $G$. Let $X$ be the set of neighbors of $x$ except for $y$ in $G$, let $Y$ be the set of neighbors of $y$ except for $x$ in $G$, let $X^{\prime}$ be the set of neighbors of $X$ except for $x$ in $G$. If $X^{\prime} \cap Y=\emptyset$, then $\left|V_{1}\right| \geq 1+|Y|+\left|X^{\prime}\right| \geq 1+(r-1)+(r-1)=2 r-1>n$, a contradiction (Note that $\left|X^{\prime}\right| \geq r-1$ ). Thus $X^{\prime} \cap Y \neq \emptyset$, that is, there exists a cycle containing $x y$ of length four in G.

Remark 2 The degree condition is optimal. Let $m, n$ be two even numbers with $n, m \geq 2$. Since $K_{n / 2, m / 2} \cup K_{n / 2, m / 2}$ is disconnected, the oriented radius (resp. diameter) of $K_{n / 2, m / 2} \cup K_{n / 2, m / 2}$ is $\infty$.

For equal bipartition $k$-regular graph, the following corollary holds.

Corollary 2 Let $G=\left(V_{1} \cup V_{2}, E\right)$ be a $k$-regular bipartite graph with $\left|V_{1}\right|=\left|V_{2}\right|=n$. If $k>n / 2$, then there exists an orientation $H$ of $G$ such that $\operatorname{rad}(H) \leq 9$.

The following theorem holds for general graphs.

Theorem 8 Let $G$ be a graph of order $n$.

(i) If there exists an integer $k \geq 2$ such that $\left|N_{k}(u)\right|>n / 2-1$ for every vertex $u$ in $G$, then $G$ has an orientation $H$ such that $\operatorname{rad}(H) \leq 4 k^{2}$ and $\operatorname{diam}(H) \leq 8 k^{2}$.

(ii) If $\delta(G)>n / 2$, then $G$ has an orientation $H$ such that $\operatorname{rad}(H) \leq 4$ and $\operatorname{diam}(H) \leq 8$.

Proof: Since methods of the proofs of $(i)$ and $(i i)$ are similar, we only prove $(i)$. For $(i)$, it suffices to show that $\operatorname{rad}(G) \leq 2 k$ and $\eta(G) \leq 2 k+1$ by Theorem 2 .

We first show $\operatorname{rad}(G) \leq 2 k$. Fix $u$ in $G$, for every $v \in V(G)$, if $v \in N_{k}[u]$, then $d(u, v) \leq k$. Suppose $v \notin N_{k}[u]$, we have $N_{k}(u) \cap N_{k}(v) \neq \emptyset$. If not, that is, $N_{k}(u) \cap N_{k}(v)=\emptyset$, then $\left|N_{k}(u)\right|+\left|N_{k}(v)\right|+2>$ $n$ (a contradiction). Thus $d(u, v) \leq 2 k$. 
Next we show $\eta(G) \leq 2 k+1$. Let $e=u v$ be any edge in $G$. If $N_{k}(u) \cap N_{k}(v)=\emptyset$, then $|V(G)| \geq$ $\left|N_{k}(u)\right|+\left|N_{k}(v)\right|+2>n$, a contradiction. Thus $N_{k}(u) \cap N_{k}(v) \neq \emptyset$. Pick $w \in N_{k}(u) \cap N_{k}(v)$, and let $P$ (resp. $Q$ ) be a path between $u$ and $w$ (resp. between $v$ and $w$ ). Then $e$ belongs a close trial $u P w Q v u$ of length $2 k+1$. Therefore, $e$ belongs a cycle of length at most $2 k+1$.

Remark 3 The above condition is almost optimal since $K_{n / 2} \cup K_{n / 2}$ is disconnected for even $n$.

Corollary 3 Let $G$ be a graph with minimum degree $\delta(G)$ and girth $g(G)$. If there exists an integer $k$ such that $k \leq g(G) / 2$ and $\delta(G)(\delta(G)-1)^{k-1}>n / 2-1$, then $G$ has an orientation $H$ such that $\operatorname{rad}(H) \leq 4 k^{2}$.

Proof: Let $k$ be an integer such that $k \leq g(G) / 2$ and $\delta(G)(\delta(G)-1)^{k-1}>n / 2-1$. For any vertex $u$ of $G$, let $1 \leq i<k$ be any integer and $x, y \in N_{i}(u)$. If $x$ and $y$ have a common neighbor $z$ in $N_{i+1}(u)$, then $G$ has a cycle of length at most $2 i<2 k \leq g(G) / 2$, a contradiction. Thus $x$ and $y$ has no common neighbor in $N_{i+1}(u)$. Therefore, $\left|N_{k}(u)\right| \geq \delta(G)(\delta(G)-1)^{k-1}>n / 2-1$. By Theorem 2 , $G$ has an orientation $H$ such that $\operatorname{rad}(H) \leq 4 k^{2}$.

\section{Upper bound for rainbow connection number}

At first, we have the following observation.

Observation 2 Let $G$ be a graph and $H$ be a spanning subgraph of $G$. Then $r c(G) \leq r c(H)$.

Proof of Theorem 4; Let $u$ be a center of $G$ and let $H_{0}$ be the trivial graph with vertex set $\{u\}$. We assert that there exists a subgraph $H_{i}$ of $G$ such that $N_{i}[u] \subseteq V\left(H_{i}\right)$ and $r c\left(H_{i}\right) \leq \sum_{j=1}^{i} \min \{2(\operatorname{rad}(G)-$ $j)+1, \eta(G)\}$.

Basic step: When $i=1$, we omit the proof since the proof of this step is similar to that of the following induction step.

Induction step: Assume that the above assertion holds for $i-1$ and $c$ is a $r c\left(H_{i-1}\right)$-rainbow coloring of $H_{i-1}$. Next we show that the above assertion holds for $i$. For any $v \in N_{i}(u)$, either $v \in V\left(H_{i-1}\right)$ or $v \in N_{G}\left(V\left(H_{i-1}\right)\right.$ since $N_{i-1}[u] \subseteq V\left(H_{i-1}\right)$. If $N_{i}(u) \subseteq V\left(H_{i-1}\right)$, then let $H_{i}=H_{i-1}$ and we are done. Thus, we suppose $N_{i}(u) \not \subset V\left(H_{i-1}\right)$ in the following.

Let $C_{1}=\left\{\alpha_{1}, \alpha_{2}, \cdots\right\}$ and $C_{2}=\left\{\beta_{1}, \beta_{2}, \cdots\right\}$ be two pools of colors, none of which are used to color $H_{i-1}$, and there exists no common color in $C_{1}$ and $C_{2}$. An edge-coloring of an $H$-ear $P=$ $\left(u_{0}, u_{1}, \cdots, u_{k}\right)$ is a symmetrical coloring if its edges are colored by $\alpha_{1}, \alpha_{2}, \cdots, \alpha_{\lceil k / 2\rceil}, \beta_{\lfloor k / 2\rfloor}, \cdots, \beta_{2}$, $\beta_{1}$ in that order or $\beta_{1}, \beta_{2}, \cdots, \beta_{\lfloor k / 2\rfloor}, \alpha_{\lceil k / 2\rceil} \cdots, \alpha_{2}, \alpha_{1}$ in that order.

Let $X=N_{i}(u) \backslash V\left(H_{i-1}\right)$ and $m=\min \{2(\operatorname{rad}(G)-i)+1, \eta(G)\}$. Pick $x_{1} \in X$, Let $y_{1}$ be a neighbor of $x_{1}$ in $H_{i-1}$ and $P_{1}$ be an optimal $\left(H_{i-1}, x_{1} y_{1}\right)$-ear. We can color $P$ symmetrically with colors $\alpha_{1}, \alpha_{2}, \cdots, \alpha_{\lceil\ell(P) / 2\rceil}, \beta_{\lfloor\ell(P) / 2\rfloor}, \cdots, \beta_{2}, \beta_{1}$. Pick $x_{2} \in X$ satisfying that all the incident edges of $x_{2}$ are not colored. Let $y_{2}$ be a neighbor of $x_{2}$ in $H_{i-1}$. If there exists an optimal $\left(H_{i-1}, x_{2} y_{2}\right)$-ear $P_{2}$ such that $P_{1}$ and $P_{2}$ are independent, then we can color $P_{2}$ symmetrically with colors $\alpha_{1}, \alpha_{2}, \cdots, \alpha_{\left\lceil\ell\left(P_{2}\right) / 2\right\rceil}, \beta_{\left\lfloor\ell\left(P_{2}\right) / 2\right\rfloor}, \ldots, \beta_{2}, \beta_{1}$. Otherwise, by Lemma 1 , there exists an optimal $\left(H_{i-1}, x_{2} y_{2}\right)$-ear $P_{2}=P_{y_{2} z_{2}}$ such that $P_{1}$ and $P_{2}$ have only one continuous common segment containing $z_{2}$, where $z_{2}$ is the other foot of $P_{2}$. Thus we can color $P_{2}$ symmetrically with colors 
$\alpha_{1}, \alpha_{2}, \cdots, \alpha_{\left\lceil\ell\left(P_{2}\right) / 2\right\rceil}, \beta_{\left\lfloor\ell\left(P_{2}\right) / 2\right\rfloor}, \ldots, \beta_{2}, \beta_{1}$ by preserving the coloring of $P_{1}$. We can pick the vertices of $X$ and color optimal $H_{i}$-ears until that for any $x \in X$, at least two incident edges of $x$ are colored. Since for any leg $e$ of $H_{i-1}, \ell(e) \leq m$ by Lemma 3, we use at most $m$ coloring in the above coloring process.

Let $H_{i}$ be the graph obtained from $H_{i-1}$ by adding all the vertices in $V(G) \backslash V\left(H_{i-1}\right)$, which have at least two new colored incident edges, and adding the new colored edges. Clearly, $N_{i}[u] \subseteq V\left(H_{i}\right)$. It is suffices to show that $H_{i}$ is rainbow connected. Let $x$ and $y$ be two distinct vertices in $H_{i}$. If $x, y \in$ $V\left(H_{i-1}\right)$, then there exists a rainbow path between $x$ and $y$ by inductive hypothesis. If exactly one of $x$ and $y$ belongs to $V\left(H_{i-1}\right)$, say $x$. Let $P$ be a symmetrical colored $H_{i-1}$-ear containing $y$, and $y^{\prime}$ be a foot of $P$. There exists a rainbow path $Q$ between $x$ and $y^{\prime}$ in $H_{i-1}$ by inductive hypothesis. Thus, $x Q y^{\prime} P y$ is a rainbow path between $x$ and $y$ in $H_{i}$.

Suppose none of $x$ and $y$ belongs to $H_{i-1}$. Let $P$ and $Q$ be symmetrical colored $H_{i-1}$-ear containing $x$ and $y$, respectively. Furthermore, let $x^{\prime}, x^{\prime \prime}$ be the feet of $P$ and $y^{\prime}, y^{\prime \prime}$ be the feet of $Q$. Without loss of generality, assume that $P$ is colored from $x^{\prime}$ to $x^{\prime \prime}$ by $\alpha_{1}, \alpha_{2}, \cdots, \alpha_{\lceil\ell(P) / 2\rceil}, \beta_{\lfloor\ell(P) / 2\rfloor}, \ldots, \beta_{2}, \beta_{1}$ in that order, and $Q$ is colored from $y^{\prime}$ to $y^{\prime \prime}$ by $\alpha_{1}, \alpha_{2}, \cdots, \alpha_{[\ell(Q) / 2\rceil}, \beta_{\lfloor\ell(Q) / 2\rfloor}, \ldots, \beta_{2}, \beta_{1}$ in that order. If $\ell\left(x^{\prime} P x\right) \leq \ell\left(y^{\prime} Q y\right)$, let $R$ be a rainbow path between $x^{\prime}$ and $y^{\prime \prime}$ in $H_{i-1}$. Then $x P x^{\prime} R y^{\prime \prime} Q y$ is a rainbow path between $x$ and $y$ in $H_{i}$. Otherwise, $\ell\left(x^{\prime} P x\right)>\ell\left(y^{\prime} Q y\right)$. Let $R$ be a rainbow path between $y^{\prime}$ and $x^{\prime \prime}$ in $H_{i-1}$. Then $y Q y^{\prime} R x^{\prime \prime} P x$ is a rainbow path between $x$ and $y$ in $H_{i}$. Thus, there exists a rainbow path between any two distinct vertices in $H_{i}$, that is, $H_{i}$ is $\left(\Sigma_{j=1}^{i} \min \{2(\operatorname{rad}(G)-j)+1, \eta(G)\}\right)$-rainbow connected. $\quad \square$ Readers can see [1] for an optimal example. The following example shows that our result is better than that of Theorem 3 .

Example 2 Let $r \geq 3, k \geq 2 r$ be two integers, and $W_{k}=C_{k} \vee K_{1}$ be an wheel, where $V\left(C_{k}\right)=$ $\left\{u_{1}, u_{2}, \ldots, u_{k}\right\}$ and $V\left(K_{1}\right)=\{u\}$. Let $H$ be the graph obtained from $W_{k}$ by inserting $r-1$ vertices between every edge $u u_{i}, 1 \leq i \leq k$. For every edge $e=x y$ of $H$, add a new vertex $v_{e}$ and new edges $v_{e} x, v_{e} y$. Denote by $G$ the resulting graph. It is easy to check that $\operatorname{rad}(G)=r, \operatorname{diam}(G)=2 r, \eta(G)=3$ and $\zeta(G)=2 r-1$. By Theorem 3 , we have $r c(G) \leq \sum_{i=1}^{r} \min \{2 i+1, \zeta(G)\} \leq r^{2}+2 r-2$. But, by Theorem 4 we have $r c(G) \leq 3 r$. On the other hand, $r c(G) \geq 2 r$ since $\operatorname{diam}(G)=2 r$.

The remaining results are similar to those in Section 3.

Theorem 9 Let $G$ be a plane graph. If the length of the boundary of every face is at most $k$, then $\operatorname{rc}(G) \leq k \operatorname{rad}(G)$.

Corollary 4 Let $G$ be a maximal plane (resp. outerplane) graph. Then $\operatorname{rc}(G) \leq 3 \mathrm{rad}(G)$.

Theorem 10 Let $G$ be a bridgeless edge-transitive graph. Then $\operatorname{rc}(G) \leq \operatorname{rad}(G) g(G)$, where $g(G)$ is the girth of $G$.

Theorem 11 Let $G=\left(V_{1} \cup V_{2}, E\right)$ be a bipartite graph with $\left|V_{1}\right|=n$ and $\left|V_{2}\right|=m$. If $d(x) \geq k>$ $\lceil m / 2\rceil$ for any $x \in V_{1}, d(y) \geq r>\lceil n / 2\rceil$ for any $y \in V_{2}$, then $r c(G) \leq 12$.

Remark 4 The degree condition is optimal. Let $m, n$ be two even numbers with $n, m \geq 2$. Since $K_{n / 2, m / 2} \cup K_{n / 2, m / 2}$ is disconnected, $\operatorname{rc}\left(K_{n / 2, m / 2} \cup K_{n / 2, m / 2}\right)=\infty$.

Corollary 5 Let $G=\left(V_{1} \cup V_{2}, E\right)$ be a k-regular bipartite graph with $\left|V_{1}\right|=\left|V_{2}\right|=n$. If $k>\lceil n / 2\rceil$, then $\operatorname{rc}(G) \leq 12$.

The following theorem holds for general graphs. 
Theorem 12 Let $G$ be a graph.

(i) If there exists an integer $k \geq 2$ such that $\left|N_{k}(u)\right|>n / 2-1$ for every vertex $u$ in $G$, then $\operatorname{rc}(G) \leq$ $4 k^{2}+2 k$.

(ii) If $\delta(G)>n / 2$, then $r c(G) \leq 6$.

Remark 5 The above condition is almost optimal since $K_{n / 2} \cup K_{n / 2}$ is disconnected for even $n$.

Corollary 6 Let $G$ be a graph with minimum degree $\delta(G)$ and girth $g(G)$. If there exists an integer $k$ such that $k<g(G) / 2$ and $\delta(G)(\delta(G)-1)^{k-1}>n / 2-1$, then then $r c(G) \leq 4 k^{2}+2 k$.

\section{Acknowledgments}

The authors are very grateful to the referees for their helpful comments and suggestions.

\section{References}

[1] M. Basavaraju, L.S. Chandran, D. Rajendraprasad, A. Ramaswamy, Rainbow connection number and radius, Graphs \& Combin. 30(2014), 275-285.

[2] J.A. Bondy, U.S.R. Murty, Graph Theory, GTM 244, Springer, 2008.

[3] S. Chakraborty, E. Fischer, A. Matsliah, R. Yuster, Hardness and algorithms for rainbow connectivity, J. Combin. Optim. 21(2011), 330-347.

[4] G. Chartrand, G.L. Johns, K.A. McKeon, P. Zhang, Rainbow connection in graphs, Math. Bohem. 133(2008), 85-98.

[5] V. Chvátal, C. Thomassen, Distances in orientations of graphs, J. Combin. Theory, Ser.B, 24(1978), 61-75.

[6] F.V. Fomin, M. Matamala, E. Prisner, I. Rapaport, AT-free graphs: Linear bounds for the oriented diameter, Discrete Appl. Math. 141(2004), 135-148.

[7] K.M. Koh, B.P. Tan, The minimum diameter of orientations of complete multipartite graphs, Graphs \& Combin. 12(1996), 333-339.

[8] K.M. Koh, E.G. Tay, Optimal orientations of products of paths and cycles, Discrete Appl. Math. 78(1997), 163-174.

[9] J.C. Konig, D.W. Krumme, E. Lazard, Diameter-preserving orientations of the torus, Networks 32(1998), 1-11.

[10] M. Krivelevich, R. Yuster, The rainbow connection of a graph is (at most) reciprocal to its minimum degree, J. Graph Theory 63(2010), 185-91.

[11] P.K. Kwok, Q. Liu, D.B. West, Oriented diameter of graphs with diameter 3, J. Combin. Theory, Ser. B, 100(2010), 265-274.

[12] H. Li, X. Li, S. Liu, The (strong) rainbow connection numbers of Cayley graphs on Abelian groups, Comput. Math. Appl. 62(11)(2011), 4082-4088.

[13] X. Li, Y. Sun, Upper bounds for the rainbow connection number of line graphs, Graphs \& Combin. (28)2012, 251-263.

[14] J.E. Mccanna, Orientations of the $n$-cube with minimum diameter, Discrete Math. 68(1988), 309-310.

[15] J. Plesník, Remarks on diameters of orientations of graphs, Acta Math. Univ. Comenian. 46/47(1985), 225-236.

[16] H. Robbins, A theorem on graphs with an application to a problem of traffic control, Amer. Math. Monthly 46(1939), 281-283.

[17] I. Schiermeyer, Rainbow connection in graphs with minimum degree three, In J. Fiala, J. Kratochvl, M. Miller, editors, Combinatorial Algorithms, Lecture Notes in Computer Science Vol.5874 (2009), 432-437. Springer Berlin/Heidelberg.

[18] L. Šoltés, Orientations of graphs minimizing the radius or the diameter, Math. Slovaca 36(1986), 289-296. 